\title{
Economic aspects of cooperation between New Zealand and the United States as international actors in the Asia-Pacific region
}

\author{
Elena Novikova $^{1}$ and Mikhail Rybalko ${ }^{1}$ \\ ${ }^{1}$ Irkutsk State University, Lenin str., 8, Irkutsk, 664025, Russia
}

\begin{abstract}
The article analyzes economic aspects of the interaction between New Zealand and the United States of America as international actors in the Asia-Pacific region. The substantiation of the importance of the Asia-Pacific region in the foreign policy of the two countries is given. We determined that the economic component is one of the most significant components of bilateral relations. Statistical data for the five-year period (2016-2021) are presented, demonstrating the evolution of economic relations between New Zealand and the United States. We established that the economic policy of the two countries is aimed at continuing intensive international cooperation and implementing a recovery strategy in 2021. We concluded that the "soft power" course used by Wellington would enable the country to achieve significant benefits even more in cooperation with other states and reach qualitatively new levels in the existing system of international relations in the Asia-Pacific region.
\end{abstract}

\section{Introduction}

The global transformations of the XXI century, the strengthening of the processes of globalization, integration, regionalization, the evolution of uneven development of regions and difficulties in ensuring their sustainable development justify the relevance of the study of regional processes in the world [1]. According to a number of experts, the processes of regionalization and globalization are changing dynamically and have a two-way influence on each other [2].

The Asia-Pacific region (APR) is one of the most dynamically developing regions of the world. It is becoming increasingly important from a strategic point of view. Trade, investment and financial flows are gradually shifting here. Integration processes are intensively unfolding in the region, the interweaving of bilateral and multilateral free trade zones is taking shape. It was in the Asia-Pacific region that the TransPacific Partnership was concluded, which became the first mega-regional trade agreement. The future of the world economy and security largely depends on the degree of openness of cooperation between the countries of this geographical region.

The beginning of the new century was marked by the expansion of the vector of New Zealand's foreign policy, namely towards the Asia-Pacific region, which was explained by the accumulation of economic, political and geostrategic growth in the countries of this region. In addition, the strengthening of New Zealand's relations with the Asia-Pacific region has peaked since the 2010 s due to the stabilization of the country's economy, which has become an impetus for investment opportunities and the improvement of New Zealand's foreign policy and the demonstration of its certain political claims in the region. Despite the fact that the country does not conduct active economic expansion like the Australian Union, it is considered one of the most stable and desirable partners in international relations in the Asia-Pacific region [3].

New Zealand and the USA are significant partners for each other. The history of diplomatic relations between the two countries dates back to the establishment of the U.S. Consular Mission in 1838 and the establishment of official diplomatic relations in 1942.

This article examines the interaction between the United States and New Zealand over the past 5 years through the prism of the economic component as one of the most significant components of bilateral relations.

\section{Results and discussion}

According to the New Zealand government, the United States ranks third in the list of sources of foreign direct investment in New Zealand (Australia and Hong Kong are leading). Thus, in 2018 , this figure amounted to $\$$ 11.3 billion. The US direct investment in New Zealand is concentrated in the manufacturing, financial and wholesale trade sectors. The space sector is an area of growth thanks to joint investments in Rocket Lab and LeoLabs and a partnership offering NASA New Zealand scholarships. More than 300 American companies have subsidiaries in New Zealand. Many

\footnotetext{
Corresponding author: e.novikova@mntk.irkutsk.ru
} 
work through local agents, and some are united in joint ventures [4].

On July 20, 2018, the United States and New Zealand held a meeting within the framework of the Trade and Investment Framework Agreement (TIFA), noting the strong and growing trade relations between the two countries and discussing the full range of bilateral issues [5].

In 2016, the United States exported $\$ 3.52$ billion to New Zealand. The main exported goods were airplanes, helicopters and/or spacecraft (US\$576 million (16.4\%)), automobiles (US\$234 million $(6.65 \%))$ and gas turbines (US\$174 million (4.93\%)).

Among other exported goods were medical instruments (US\$90 million $(2.56 \%)$ ), medicines (US\$50 million (1.42\%)), aircraft parts (US\$79.7 million $(2.26 \%)$ ), tractors (US\$39.3 million (1.12\%)), trucks (US\$29.3 million $(0.83 \%)$ ), laboratory reagents (US\$25.7 million $(0.73 \%)$ ), animal feed (US\$44.4 million $(1.26 \%)$ ), petroleum coke (36.7 million US dollars (1.04\%)) [6].

In 2017 , the US exports were worth 3.72 billion US dollars to New Zealand. The main exported goods were airplanes, helicopters and/or spacecraft (US\$415 million (11.1\%)), automobiles (US\$316 million $(8.5 \%))$ and gas turbines (US\$203 million (5.47\%)).

Among other exported goods there were medical instruments (94 million US dollars $(2.53 \%)$ ), medicines (49.7 million US dollars $(1.34 \%)$ ), aircraft parts (79.7 million US dollars $(2.14 \%)$ ), tractors (50.6 million US dollars $(1.36 \%))$, trucks (13.4 million US dollars $(0.36 \%))$, laboratory reagents $(26.5$ million US dollars $(0.71 \%))$, animal feed (42.5 million US dollars $(1.14 \%))$, petroleum coke (27.4 million US dollars $(0.74 \%))[6]$.

In 2018, the United States of America exports increased to 3.76 billion US dollars to New Zealand. The main exports were airplanes, helicopters and/or spacecraft (326 million US dollars (8.66\%)), automobiles (288 million US dollars $(7.65 \%)$ ) and gas turbines (257 million US dollars $(6.83 \%))$.

Other exported goods were medical instruments (US\$99.9 million (2.65\%)), medicines (US\$47.7 million (1.27\%)), aircraft parts (US\$87.9 million $(2.34 \%)$ ), tractors (US\$60.7 million $(1.61 \%)$ ), trucks (US\$42.1 million $(1.12 \%)$ ), laboratory reagents (US\$33.5 million $(0.89 \%)$ ), animal feed (US\$45.5 million $(1.21 \%)$ ), petroleum coke (36.8 million US dollars $(0.98 \%))$ [6].

In 2019, American exports to New Zealand amounted to 3.65 billion US dollars. The main commodities were gas turbines (US\$349 million $(9.56 \%)$ ), automobiles (US\$262 million $(7.17 \%)$ ) and medical instruments (US\$106 million (2.9\%)).

Among other exported goods were medical instruments (106 million US dollars $(2.9 \%))$, medicines (47.7 million US dollars (1.27\%)), aircraft parts (72.1 million US dollars $(1.98 \%))$, tractors (30.9 million US dollars $(0.85 \%))$, trucks $(60$ million US dollars $(1.65 \%))$, laboratory reagents (41.3 million US dollars $(1.13 \%))$, animal feed (48.7 million US dollars
(1.34\%)), petroleum coke ( US\$30.1 million (0.83\%)) [6].

\section{US Exports to New Zealand (2016-2019)}

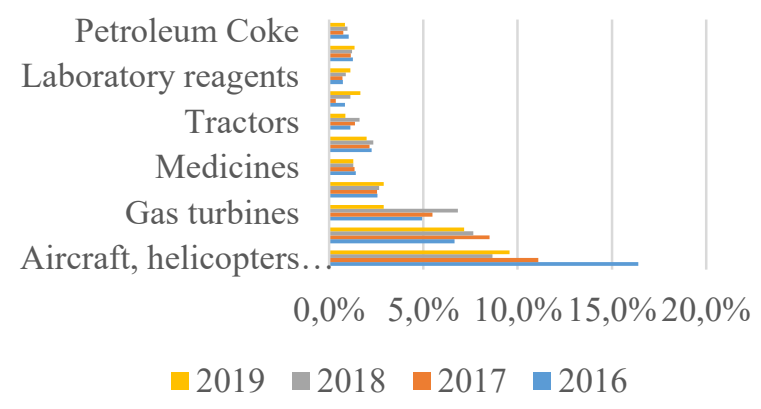

Figure 1. US Exports to New Zealand (2016-2019)

In 2016, New Zealand exports reached US\$ 3.76 billion to the United States. The main products exported from New Zealand to the United States were frozen cattle meat (US\$843 million (22.4\%)), wine (US\$356 million $(9.49 \%)$ ), whey (US\$259 million $(6.9 \%)$ ), sheep and goat meat (US\$191 million $(5.08 \%)$ ), casein (US\$207 million $(5.5 \%)$ ), lumber (US\$138 million $(3.67 \%)$ ), therapeutic devices (120 million US dollars $(3.19 \%))$, apples and pears (83.9 million US dollars $(2.24 \%)$ ), water-soluble proteins (81.8 million US dollars $(2.18 \%))$, harvesting equipment (45.3 million US dollars (1.21\%)).

In 2017, New Zealand's exports amounted to 3.84 billion US dollars. The main products were frozen cattle meat (856 million US dollars $(22.3 \%)$ ), wine (381 million US dollars $(9.92 \%)$ ), sheep and goat meat (254 million US dollars $(6.61 \%)$ ), whey (232 million US dollars $(6.06 \%)$ ), casein (213 million US dollars $(5.55 \%))$, lumber (139 million US dollars $(3.63 \%))$, therapeutic devices (120 million US dollars (3.13\%)), apples and pears (68 million US dollars $(1.77 \%))$, water-soluble proteins (59 million US dollars (1.54\%)), harvesting equipment (35.4 million US dollars $(0.92 \%))$.

In 2018, New Zealand exports to the United States amounted to 3.88 billion US dollars. These are frozen cattle meat (US\$821 million (21.2\%)), wine (US\$381 million (9.84\%)), sheep and goat meat (US\$300 million $(7.73 \%)$ ), whey (US\$211 million $(5.45 \%)$ ), casein (US\$138 million (3.56\%)), lumber (US\$123 million (3.18\%)), therapeutic devices (US\$106 million $(2.73 \%))$, apples and pears (64.7 million US dollars $(1.67 \%))$, water-soluble proteins (32.5 million US dollars $(0.84 \%))$, harvesting equipment (31.3 million US dollars $(0.81 \%))$.

In 2019, New Zealand exports increased to 3.78 billion US dollars to the United States of America. The exported goods included frozen cattle meat (US\$601 million (15.9\%)), wine (US\$413 million (10.9\%)), sheep and goat meat (US\$281 million $(7.44 \%)$ ), whey (US\$216 million $(5.72 \%)$ ), casein (US\$144 million $(3.82 \%)$ ), lumber (US\$138 million (3.64\%)), therapeutic devices (US\$109 million $(2.88 \%)$ ), apples and pears (55 million US dollars $(1.46 \%)$ ), water- 
soluble proteins (34.7 million US dollars (0.92\%)), harvesting equipment (34.7 million US dollars USA $(0.92 \%))$ [6].

New Zealand Exports to the US (20162019)

Cleaning equipment

Water-soluble..

Apples and pears

Therapeutic devices

Lumber

Casein

Sheep and goat.

Whey

Wine

Frozen cattle meat

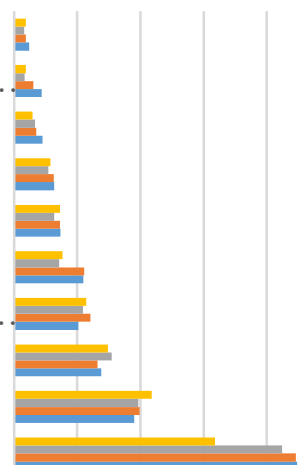

$0,0 \%, 0 \% 0,0 \% 5,020,025,0 \%$

$\square 2019 \square 2018 \square 2017 \quad 2016$

Figure 2. New Zealand Exports to the US (20162019)

Thus, according to these statistics, the results of trade volumes and growth rates for the main groups of goods for 2016-2019 were as follows (Table 1):

Table 1. Trade volumes and growth rates (2016-2019)

\begin{tabular}{|c|c|c|c|}
\hline \multicolumn{4}{|c|}{ US Exports to New Zealand } \\
\hline Year & Goods & $\begin{array}{l}\text { Trade } \\
\text { volume } \\
\text { (USD } \\
\text { million) }\end{array}$ & $\begin{array}{l}\text { Trade } \\
\text { growth } \\
\text { rate }(\%)\end{array}$ \\
\hline \multirow{11}{*}{$\begin{array}{l}2016- \\
2017\end{array}$} & $\begin{array}{l}\text { airplanes, } \\
\text { helicopters and/or } \\
\text { spacecraft }\end{array}$ & -162 & -28 \\
\hline & automobiles & +81.9 & +35 \\
\hline & gas turbines & +29.7 & +17.1 \\
\hline & $\begin{array}{l}\text { medical } \\
\text { instruments }\end{array}$ & +3.97 & +4.41 \\
\hline & medicines aircraft & -234 & -0.47 \\
\hline & parts & -4.36 & -0.0055 \\
\hline & tractors & +11.3 & +28.7 \\
\hline & trucks & -15.9 & -54.4 \\
\hline & $\begin{array}{l}\text { laboratory } \\
\text { reagents animal }\end{array}$ & +0.875 & +3.41 \\
\hline & feed & -1.88 & -4.23 \\
\hline & petroleum coke & -9.26 & -25.2 \\
\hline \multirow{5}{*}{$\begin{array}{l}2017- \\
2018\end{array}$} & $\begin{array}{l}\text { airplanes, } \\
\text { helicopters and/or } \\
\text { spacecraft }\end{array}$ & -88.8 & -21.4 \\
\hline & automobiles & -28.1 & 8.89 \\
\hline & gas turbines & +53.5 & +26.3 \\
\hline & $\begin{array}{l}\text { medical } \\
\text { instruments }\end{array}$ & +5.87 & +6.25 \\
\hline & medicines aircraft & -2.02 & -4.06 \\
\hline
\end{tabular}

\begin{tabular}{|c|c|c|c|}
\hline & parts & +8.24 & +10.3 \\
\hline & tractors & +10.1 & +20 \\
\hline & trucks & +28.8 & +216 \\
\hline & $\begin{array}{l}\text { laboratory } \\
\text { reagents animal }\end{array}$ & +6.94 & +26.1 \\
\hline & feed & +2.98 & +7.02 \\
\hline & petroleum coke & +9.35 & +34.1 \\
\hline \multirow{11}{*}{$\begin{array}{l}2018- \\
2019\end{array}$} & $\begin{array}{l}\text { airplanes, } \\
\text { helicopters and/or } \\
\text { spacecraft }\end{array}$ & -243 & -74.5 \\
\hline & automobiles & -26.4 & -9.17 \\
\hline & gas turbines & +91.8 & +35.7 \\
\hline & $\begin{array}{l}\text { medical } \\
\text { instruments }\end{array}$ & +6.02 & +6.03 \\
\hline & medicines aircraft & -6.33 & -13.3 \\
\hline & parts & -15.8 & -18 \\
\hline & tractors & -29.9 & -49.2 \\
\hline & trucks & +17.9 & +42.4 \\
\hline & $\begin{array}{l}\text { laboratory } \\
\text { reagents animal }\end{array}$ & +7.84 & +23.4 \\
\hline & feed & +3.25 & +7.16 \\
\hline & petroleum coke & -6.64 & -18.1 \\
\hline \multicolumn{4}{|c|}{ Exports to US } \\
\hline \multirow{10}{*}{$\begin{array}{l}2016- \\
2017\end{array}$} & frozen cattle meat & +13.8 & +1.64 \\
\hline & wine & +24.4 & +6.84 \\
\hline & whey & -26.7 & -10.3 \\
\hline & $\begin{array}{l}\text { sheep and goat } \\
\text { meat }\end{array}$ & +63.1 & +33.1 \\
\hline & casein & +6.38 & +3.09 \\
\hline & lumber & +1.63 & +1.18 \\
\hline & $\begin{array}{l}\text { therapeutic } \\
\text { devices }\end{array}$ & +0.195 & +0.16 \\
\hline & $\begin{array}{l}\text { apples and pears } \\
\text { water- }\end{array}$ & -16 & -19 \\
\hline & soluble proteins & -22.9 & -27.9 \\
\hline & $\begin{array}{l}\text { harvesting } \\
\text { equipment }\end{array}$ & -9.9 & -21.8 \\
\hline \multirow{10}{*}{$\begin{array}{l}2017- \\
2018\end{array}$} & frozen cattle meat & -35.3 & -4.12 \\
\hline & wine & +0.713 & +0.19 \\
\hline & whey & -21.1 & -9.07 \\
\hline & $\begin{array}{l}\text { sheep and goat } \\
\text { meat }\end{array}$ & +46 & +18.1 \\
\hline & casein & -74.9 & -35.2 \\
\hline & lumber & -16.1 & -11.5 \\
\hline & $\begin{array}{l}\text { therapeutic } \\
\text { devices }\end{array}$ & -14.4 & -12 \\
\hline & $\begin{array}{l}\text { apples and pears } \\
\text { water- }\end{array}$ & -3.32 & -4.88 \\
\hline & soluble proteins & -26.5 & -44.9 \\
\hline & $\begin{array}{l}\text { harvesting } \\
\text { equipment }\end{array}$ & -4.13 & -11.7 \\
\hline \multirow{7}{*}{$\begin{array}{l}2018- \\
2019\end{array}$} & frozen cattle meat & -220 & -26.8 \\
\hline & wine & +32 & +8.38 \\
\hline & whey & +4.52 & +2.14 \\
\hline & $\begin{array}{l}\text { sheep and goat } \\
\text { meat }\end{array}$ & -19 & -6.34 \\
\hline & casein & +6.23 & +4.51 \\
\hline & lumber & +14.4 & +11.7 \\
\hline & $\begin{array}{l}\text { therapeutic } \\
\text { devices }\end{array}$ & +3.1 & +2.94 \\
\hline
\end{tabular}




\begin{tabular}{|l|l|l|l|}
\hline & $\begin{array}{l}\text { apples and pears } \\
\text { water- }\end{array}$ & -9.67 & -15 \\
\cline { 2 - 4 } & soluble proteins & +2.23 & +6.86 \\
\cline { 2 - 4 } & $\begin{array}{l}\text { harvesting } \\
\text { equipment }\end{array}$ & +3.41 & +10.9 \\
\hline
\end{tabular}

According to the World Bank, the global economy shrank by $4.3 \%$ in 2020 due to CAVID-19, which was the worst recession since World War II. Most significant consequences were for those countries that are most dependent on world trade, tourism, commodity exports and external financing. Trade in agricultural products of particular importance to New Zealand was less affected, declining by only $5 \%$ in the second quarter of 2020 against a total decline of $21 \%$ in all trade. Due to restrictions on movement and visiting public places, New Zealand imports have been steadily declining in 2020 [7].

Table 2. Trade volumes, 2020-2021

\begin{tabular}{|c|c|c|c|c|c|c|c|}
\hline \multicolumn{8}{|c|}{ To US (2020) } \\
\hline Aug & Jul & Jun20 & $\mathrm{Ma}$ & Apr & Mar & $\mathrm{Fe}$ & Jan \\
\hline 2020 & $\begin{array}{l}20 \\
20\end{array}$ & 20 & $\begin{array}{l}\mathrm{y} \\
20 \\
20\end{array}$ & $\begin{array}{l}202 \\
0\end{array}$ & $\begin{array}{l}\text { ch } \\
202 \\
0\end{array}$ & $\begin{array}{l}\mathrm{b} \\
20 \\
20\end{array}$ & $\begin{array}{l}20 \\
20\end{array}$ \\
\hline 629 & $\begin{array}{l}53 \\
9\end{array}$ & 587 & $\begin{array}{l}54 \\
3\end{array}$ & 555 & 623 & $\begin{array}{l}55 \\
3\end{array}$ & $\begin{array}{l}48 \\
9\end{array}$ \\
\hline $\begin{array}{l}\text { Aug20 } \\
21\end{array}$ & $\begin{array}{l}\text { Jul } \\
20 \\
21\end{array}$ & $\begin{array}{l}\text { Jun } \\
2021\end{array}$ & $\begin{array}{l}\mathrm{Ma} \\
\mathrm{y} \\
20 \\
21\end{array}$ & $\begin{array}{l}\text { Apr } \\
202 \\
1\end{array}$ & $\begin{array}{l}\text { Mar } \\
\text { ch } \\
202 \\
1\end{array}$ & $\begin{array}{l}\mathrm{Fe} \\
\mathrm{b} \\
20 \\
21\end{array}$ & $\begin{array}{l}\text { Jan } \\
20 \\
21\end{array}$ \\
\hline 559 & $\begin{array}{l}63 \\
0\end{array}$ & 570 & $\begin{array}{l}60 \\
1\end{array}$ & $\begin{array}{l}546 \\
, 8\end{array}$ & 570 & $\begin{array}{l}49 \\
4\end{array}$ & $\begin{array}{l}40 \\
7\end{array}$ \\
\hline \multicolumn{8}{|c|}{ US Exports to New Zealand \$ CWA } \\
\hline $\begin{array}{l}\text { Aug20 } \\
20\end{array}$ & $\begin{array}{l}\text { Jul } \\
20 \\
20\end{array}$ & $\begin{array}{l}\text { Jun20 } \\
20\end{array}$ & $\begin{array}{l}\text { Ma } \\
\mathrm{y} \\
20 \\
20\end{array}$ & $\begin{array}{l}\text { Apr } \\
202 \\
0\end{array}$ & $\begin{array}{l}\text { Mar } \\
\text { ch } \\
202 \\
0\end{array}$ & $\begin{array}{l}\mathrm{Fe} \\
\mathrm{b} \\
20 \\
20\end{array}$ & $\begin{array}{l}\text { Jan } \\
20 \\
20\end{array}$ \\
\hline 448 & $\begin{array}{l}45 \\
4\end{array}$ & 372 & $\begin{array}{l}40 \\
6\end{array}$ & 398 & 585 & - & $\begin{array}{l}47 \\
7\end{array}$ \\
\hline $\begin{array}{l}\text { Aug20 } \\
21\end{array}$ & $\begin{array}{l}\text { Jul } \\
20 \\
21\end{array}$ & $\begin{array}{l}\text { Jun } \\
2021\end{array}$ & $\begin{array}{l}\mathrm{Ma} \\
\mathrm{y} \\
20 \\
21\end{array}$ & $\begin{array}{l}\text { Apr } \\
202 \\
1\end{array}$ & $\begin{array}{l}\text { Mar } \\
\text { ch } \\
202 \\
1\end{array}$ & $\begin{array}{l}\mathrm{Fe} \\
\mathrm{b} \\
20 \\
21\end{array}$ & $\begin{array}{l}\text { Jan } \\
20 \\
21\end{array}$ \\
\hline 513 & $\begin{array}{l}52 \\
5\end{array}$ & 525 & $\begin{array}{l}54 \\
1\end{array}$ & 468 & 501 & $\begin{array}{l}35 \\
9\end{array}$ & $\begin{array}{l}39 \\
5\end{array}$ \\
\hline
\end{tabular}

Source: Stats.NZ

https://www.stats.govt.nz/publications?filters=Econom y\&start $=108$

\section{Conclusions}

Based on the consequences of the pandemic, in June 2020, the New Zealand Government announced a "three-component trade recovery strategy", which includes supporting exporters, activating the architecture of international trade and updating key trade relations $[8,9]$.

Joe Biden, who came to power in 2021, along with the continuation of the trade confrontation with China, chose a policy of rapprochement with American allies, including New Zealand. Biden's "Invest in All of America" platform includes guarantees of a fair distribution of funds with an emphasis on women and communities of color receiving a fair share of investment dollars. All of this could provide New Zealand with an opportunity to work with the U.S. on issues that are central to the U.S. "Trade for All" agenda. This trade policy is also reflected in New Zealand's policy, which is aimed at continuing intensive international cooperation and implementing a recovery strategy in 2021.

Thus, the strategy built by the New Zealand Government in the Asia-Pacific region, based on mutual respect with partner States, raised the country's status to the level of a global player and strengthened the state's position as a reliable partner in the AsiaPacific region and in the international arena as a whole, including building a constructive dialogue with the United States. The chosen course of using "soft power" will allow New Zealand to achieve significant benefits even more in cooperation with other states and reach qualitatively new levels in the existing system of international relations in the Asia-Pacific region.

\section{References}

1. O.V. Kuznetsova, Regional Studies in the USA, 6 (2020)

2. E.V. Kremnyov, E.V. Lesnikovskaya, Ojkumena. Regional Research, 3, 65 (2019), doi: 10.24866/1998-6785/2019-3/65-75

3. E.V. Drobot, M.L. Rybalko, Ekonomicheskie otnosheniya, $10 \quad$ (1), $30 \quad$ (2020), doi: 10.18334/eo.10.1.41340

4. B. Vaughn, New Zealand: Background and Bilateral Relations with the United States, 13 (2011)

5. United States and New Zealand Meet Under Trade and Investment Framework Agreement (2018). URL: https://ustr.gov/about-us/policy-offices/pressoffice/press-releases/2018/july/united-states-andnew-zealand-meet (Accessed: 18.09.2021)

6. United States / New Zealand. URL: https://oec.world/en/profile/bilateral-

country/usa/partner/nzl?dateAvailableSelectorCoun try1=exportDateCountry1 Available7\&dynamicBila teralTradeSelector=year2019\&measureBilateralTra deSelector=vizValueOption 1 \&serviceCountry2Sele ctor=serviceYear2 13 (Accessed: 15.09.2021)

7. Trade shows signs of rebound from COVID-19 recovery still uncertain (2020). URL: https://www.wto.org/english/news_e/pres20_e/pr86 2_e.htm (Accessed: 23.09.2021)

8. D. Parker. Trade Strategy for the recovery from the impacts of COVID-19 (2020). URL: https://www.beehive.govt.nz/speech/trade-strategyrecovery-impacts-covid-1 (Accessed: 25.09.2021)

9. T. Epps, N. Swan, International Trade 2020. Trends \& insights, 7 (2020) 